BIO Web of Conferences 2, 03004 (2014)

DOI: $10.1051 /$ bioconf/ 20140203004

C) Owned by the authors, published by EDP Sciences, 2014

\title{
Analyses of volatile organic compounds and refractory organic residues coming from the heating of interstellar ice analogues
}

Grégoire Danger $^{1 *}$, Fabrice Duvernay ${ }^{1}$, Patrice Theulé ${ }^{1}$, Fabien Borget ${ }^{1}$, Pierre de Marcellus ${ }^{2}$, F-R. Orthous-Daunay ${ }^{4}$, Véronique Vuitton ${ }^{4}$, Louis le Sergeant d'Hendecourt ${ }^{2,3}$, Roland Thissen ${ }^{4}$, Thierry Chiavassa $^{1}$

${ }^{1}$ Groupe Astrochimie de l'équipe "Spectrométries et Dynamique Moléculaire", laboratoire "Physique des Interactions Ioniques et Moléculaires", UMR CNRS 7345, Université d'Aixmarseille, Centre Saint-Jérôme, case 252, Marseille, France. http://sites.univprovence.fr/piim/spip.php?rubrique93

${ }^{2}$ Univ Paris-Sud, “Astrochimie et Origines”, Institut d'Astrophysique Spatiale, UMR 8617, F-91405 Orsay, France.

${ }^{3}$ CNRS, F-91405 Orsay, France.

${ }^{4}$ Institut de Planétologie et d'Astrophysique de Grenoble IPAG/PLANETO, Bâtiment D de physique - BP 5338041 Grenoble cedex 9 France.

* Corresponding author: gregoire.danger@univ-amu.fr

\begin{abstract}
We use Very High Resolution Mass Spectrometry for analyses of the soluble fraction of yellow stuff produced in laboratory. Their analyses show that they are composed of molecules with high molecular weight $(\mathrm{m} / \mathrm{z}>4000)$. Fragmentations suggest that grafted molecules constitute a part of them. Hexamethylenetetramine derivatives have also been detected. First results and further analytical developments will be presented to facilitate the understanding of the residue composition and of its chemical evolution. Furthermore, we present for the first time the concept of the VAHIIA project which concerns the analysis of volatiles coming from the heating of interstellar ice analogues.
\end{abstract}

Understand the chemical evolution of organic matter in astrophysical environments gives us clues about the chemical composition of organic matter that may have seeded the terrestrial planets, and later be at the origin of biochemical systems on Earth. Organic matter in dense molecular clouds form ice mantles on the surface of interstellar grains that will evolve and which may be found later in planetary systems. Throughout this evolution, new and more complex molecules are formed through various energetic processes, including UV irradiation and thermal effects. Small bodies of the planetary system (asteroids and comets) will eventually serve as a reservoir for this organic matter, and be vectors for delivering it to the surface of terrestrial planets such as the primitive Earth. There is probably a link, therefore, between molecules in meteorites or cometary grains, and molecules present in the interstellar grains of dense molecular cloud, and this is these relationships that our laboratory experiments try to establish, simulating the process of evolution of interstellar ices or interstellar grains. 
This contribution focuses on two aspects of our work, which relate to refractory residue analysis formed from UV irradiation of ice analogues, the RAHIIA project, and for the other, a new project that will be developed during 2013 and aims to analyse volatile organic compounds coming from the heating of such analogues, the VAHIIA project (ANR-12-JS08-0001-01 2013-2016). Understanding the formation of refractory residues, commonly called "Yellow Stuff" is an important step to establish what kind of organic matter could be available within interplanetary objects such as comets or asteroids, and therefore available for the development of a prebiotic chemistry on the surface of terrestrial planets. We present here the first results obtained by high resolution mass spectrometry (LTQ-Orbitrap) of these residues. These analyses provide the characterization of the whole residue, and show that these residues are composed of molecules of high molecular weight $(\mathrm{m} / \mathrm{z}>4000)$, and have an average elemental compositions $\mathrm{H} / \mathrm{C}=1.5, \mathrm{~N} / \mathrm{C}=0.4, \mathrm{O} / \mathrm{C}=0.4$. Next to this global approach, this kind of analysis allows searching for specific molecules. We identified various derivatives of the hexamethylenetetramine (HMT), which confirms previous infrared spectroscopy observations. We consider these residue as the first state of the organic matter that could be present in these astrophysical environments. These residues are then submitted to various physical and chemical alterations, which lead them to evolve toward the organic matter observed in cometary grains and meteorites. The second project that will be developed during 2013, concerns the analysis of volatiles coming from the heating of these interstellar ice analogues, the VAHIIA project. This project will bring more information about the molecules that sublimate when interstellar (e.g. hot cores) or cometary ice (Rosetta mission) warm, and will also give a better understanding of the chemical processes leading to the formation of refractory residues, since a large part of molecules sublimating during the ice warming takes a part in the residue formation. 